\title{
Controlling the helicity of magnetic skyrmions in a $\beta$-Mn-type high-temperature chiral magnet
}

\author{
K. Karube, ${ }^{1}$ K. Shibata,${ }^{1}$ J. S. White,${ }^{2}$ T. Koretsune,${ }^{3}$ X. Z. Yu, ${ }^{1}$ Y. Tokunaga, ${ }^{4}$ \\ H. M. Rønnow, ${ }^{5}$ R. Arita, ${ }^{1,6}$ T. Arima, ${ }^{1,4}$ Y. Tokura, ${ }^{1,6}$ and Y. Taguchi ${ }^{1}$ \\ ${ }^{1}$ RIKEN Center for Emergent Matter Science (CEMS), Wako 351-0198, Japan \\ ${ }^{2}$ Laboratory for Neutron Scattering and Imaging (LNS), Paul Scherrer Institute (PSI), CH-5232 Villigen, Switzerland \\ ${ }^{3}$ Department of Physics, Tohoku University, Sendai 980-8578, Japan \\ ${ }^{4}$ Department of Advanced Materials Science, University of Tokyo, Kashiwa 277-8561, Japan \\ ${ }^{5}$ Laboratory for Quantum Magnetism (LQM), Institute of Physics, École Polytechnique Fédérale de Lausanne (EPFL), \\ CH-1015 Lausanne, Switzerland \\ ${ }^{6}$ Department of Applied Physics, University of Tokyo, Bunkyo-ku 113-8656, Japan
}

(Received 27 July 2018; published 11 October 2018)

\begin{abstract}
Magnetic helices and skyrmions in noncentrosymmetric magnets are representative examples of chiral spin textures in solids. Their spin swirling direction, often termed as the magnetic helicity and defined as either left handed or right handed, is uniquely determined by the Dzyaloshinskii-Moriya interaction (DMI) in fixed chirality host crystals. Thus far, there have been relatively few investigations of the DMI in metallic magnets as compared with insulating counterparts. Here, we focus on the metallic magnets $\mathrm{Co}_{8-x} \mathrm{Fe}_{x} \mathrm{Zn}_{8} \mathrm{Mn}_{4}(0 \leqslant x \leqslant 4.5)$ with a $\beta$-Mn-type chiral structure and find that, as $x$ varies under a fixed crystal chirality, a reversal of magnetic helicity occurs at $x_{\mathrm{c}} \sim 2.7$. This experimental result is supported by a theory based on first-principles electronic structure calculations, demonstrating the DMI to depend critically on the electron band filling. Thus by composition tuning our work shows the sign change of the DMI with respect to a fixed crystal chirality to be a universal feature of metallic chiral magnets.
\end{abstract}

DOI: 10.1103/PhysRevB.98.155120

\section{INTRODUCTION}

Chirality of matter, described as left handed or right handed, is an important concept that permeates diverse areas of science, ranging from physics and chemistry to biology. In condensed-matter physics, chirality can be displayed by both crystal structures and symmetries, and magnetic spin textures. One example is magnetic helices in structurally chiral materials where magnetic moments gradually rotate in a clockwise $(\mathrm{CW})$ or counterclockwise (CCW) manner along a helical axis. A further related example is magnetic skyrmions, vortexlike topological spin textures [1,2]. Thus far, these magnetic textures have been extensively studied for several classes of chiral magnets [3-11]. Among them, skyrmions exhibit various intriguing properties, and are anticipated to be applied to magnetic memory devices since they can be treated as particles and can be driven by a low current density [12-15].

A microscopic mechanism producing helices and skyrmions in these chiral magnets is a competition between ferromagnetic exchange interaction $J$ and Dzyaloshinskii-Moriya interaction (DMI) $D$, the latter of which arises from the relativistic spin-orbit interaction. The effect of the DMI is to gradually twist otherwise ferromagnetically coupled magnetic moments to form a helix with a long period (10-100 $\mathrm{nm})$ as described by a magnetic wave vector $\boldsymbol{q}$. Here, $|\boldsymbol{q}|(=q)$ is proportional to $D / J$, and the helical periodicity $\lambda$ is given by $\lambda=2 \pi / q$. Depending on the swirling direction of the magnetic moments, magnetic helicity is defined as either left handed or right handed, as shown in Figs. 1(c) and 1(d). Near the helimagnetic transition temperature $T_{\mathrm{c}}$, magnetic fields induce a triangular-lattice skyrmion crystal (SkX), which is often described as a triple- $\boldsymbol{q}$ structure with the $\boldsymbol{q}$ vectors displaying mutual $120^{\circ}$ angles. Figures 1(e) and 1(f) show that the swirling direction of the in-plane magnetic moments in the skyrmions is either $\mathrm{CW}$ or $\mathrm{CCW}$, with the helicity of the magnetic helices and skyrmions being one-to-one correspondent with each other as illustrated in Figs. 1(c)-1(f). It is established that the magnetic helicity is uniquely determined by the sign of $D$ [16-22], while the size of a skyrmion is governed by the magnitude of $D$. Therefore, for a versatile use of skyrmions in applications it is important to have a control over $D$.

Previous experimental studies revealed that the relative sign of $D$ can be different within B20-type compounds with the space group of $P 2_{1} 3$, as, for example, between $\mathrm{Mn}_{1-x} \mathrm{Fe}_{x} \mathrm{Si}(0 \leqslant x \leqslant 0.11)$ and $\mathrm{Fe}_{1-x} \mathrm{Co}_{x} \mathrm{Si}(0 \leqslant x \leqslant$ $0.5)$ [16-18]. Remarkably, a continuous variation in $D$ as a function of chemical composition and even its sign reversal were found in $\mathrm{Mn}_{1-x} \mathrm{Fe}_{x} \mathrm{Ge}[19,20]$; at a critical composition $x_{\mathrm{c}} \sim 0.8, D$ is almost zero and the magnetic ground state is nearly ferromagnetic $(q \sim 0)$ rather than helimagnetic. In $\mathrm{Fe}_{1-x} \mathrm{Co}_{x} \mathrm{Ge}$ [21], the sign reversal of $D$ is reported to occur around $x_{\mathrm{c}} \sim 0.6$. Theoretically, it was a challenge to describe the DMI on the long-period, smoothly varying magnetization textures, particularly in the B20-type metallic magnets. Recently, however, there has been significant progress in the theoretical approaches, such as those based on the total energy of spin spirals [23-25], tight-binding representation [26], Berry phase $[25,27,28]$, multiscale approach with energy 


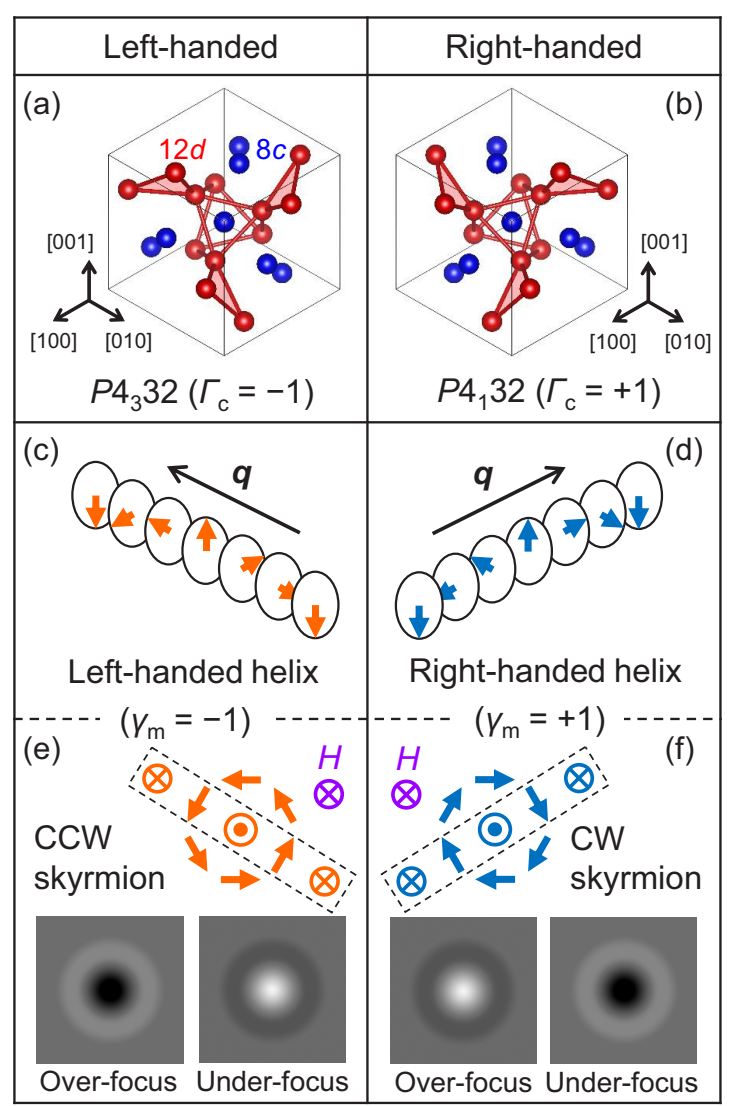

FIG. 1. (a),(b) Schematics of $\beta$-Mn-type chiral crystal structures as viewed along the [111] direction. Two enantiomers with space group $P 4_{3} 32$ and $P 4_{1} 32$ are shown. Their crystal chirality is defined as left handed $\left(\Gamma_{\mathrm{c}}=-1\right)$ and right handed $\left(\Gamma_{\mathrm{c}}=+1\right)$, respectively. Blue and red circles represent $8 c$ and $12 d$ Wickoff sites, respectively. The network of $12 d$ sites forms a windmill structure with cornersharing triangles. (c),(d) Schematic configurations of magnetic moments for left-handed helix $\left(\gamma_{\mathrm{m}}=-1\right)$ and right-handed helix $\left(\gamma_{\mathrm{m}}=\right.$ +1 ). (e),(f) Schematic configurations of in-plane magnetic moments of skyrmions: counterclockwise (CCW) skyrmion and clockwise (CW) skyrmion. Here, an external magnetic field is assumed to be applied into the page, and the magnetic moments at the periphery and the core of skyrmions are pointing into the page and out of the page, respectively. The magnetic configurations of the $\mathrm{CCW}$ and $\mathrm{CW}$ skyrmions along a radial direction indicated by dashed rectangles are equivalent to left-handed and right-handed helices as shown in (c) and (d), respectively. The corresponding overfocused and underfocused Lorentz transmission electron microscope images are also displayed schematically.

mapping [29], spin susceptibility [30], or spin current [31] formulations. Promisingly, the aforementioned variation in DMI accompanied by sign reversal can be understood in terms of a change in band filling of $3 d$ orbitals [25] within a complex electronic structure with multiple band-anticrossing points [30,31]. Nevertheless, it remains elusive whether such a sign change in DMI may take place universally in other metallic magnets with different chiral crystal structures and moreover with higher $T_{\mathrm{c}}$ 's that lie closer to room temperature. In this paper, we report experimental and theoretical investigations of $\beta$-Mn-type Co-Fe-Zn-Mn alloys, and reveal how the DMI varies as a function of band filling in a class of metallic chiral magnets very much distinct to the B20-type compounds.

Co-Zn-Mn alloys crystallize in a $\beta$-Mn-type chiral cubic structure with a space group $P 4_{1} 32$ or $P 4_{3} 32$, where 20 atoms per unit cell are distributed over two Wyckoff sites ( $8 c$ and 12d) [32,33], as illustrated in Figs. 1(a) and 1(b). Recently, Co-Zn-Mn alloys have been found to host DMI-induced helices and skyrmions at and above room temperature [34]. Mnfree $\mathrm{Co}_{10} \mathrm{Zn}_{10}$ shows a helimagnetic state with $\lambda \sim 185 \mathrm{~nm}$ below $T_{\mathrm{c}} \sim 460 \mathrm{~K}$, and both $\lambda$ and $T_{\mathrm{c}}$ decrease as partial substitution of $\mathrm{Mn}$ proceeds. In $\mathrm{Co}_{8} \mathrm{Zn}_{8} \mathrm{Mn}_{4}(\lambda \sim 120 \mathrm{~nm}$, $\left.T_{\mathrm{c}} \sim 300 \mathrm{~K}\right)$, a SkX state forms at room temperature under magnetic fields as an equilibrium state, and over a much wider temperature and field region as a metastable state [35]. Further increasing the Mn concentration suppresses the helimagnetic state, and instead promotes a spin glass state, probably due to frustrated antiferromagnetic correlations of $\mathrm{Mn}$ moments [36]. While it was reported that $D$ in $\mathrm{Co}_{8} \mathrm{Zn}_{8} \mathrm{Mn}_{4}$ and $\mathrm{Co}_{9} \mathrm{Zn}_{9} \mathrm{Mn}_{2}$ are several times smaller than that in $\mathrm{FeGe}$ [37], no systematic investigation of the DMI as a function of band filling has been performed yet.

Here, we target the Fe-doped system $\mathrm{Co}_{8-x} \mathrm{Fe}_{x} \mathrm{Zn}_{8} \mathrm{Mn}_{4}$, where a single $\mathrm{Fe}$ substitution for a Co corresponds to removing one electron per unit cell from the system. Bulk samples were successfully synthesized while keeping the $\beta$-Mn-type structure and spatial homogeneity of the element distribution up to $x=4.5$. To clarify the magnetic and crystal structures, we have performed measurements of magnetization, ac magnetic susceptibility, and small-angle neutron scattering (SANS) on the bulk samples, as well as convergent-beam electron diffraction (CBED) and Lorentz transmission electron microscopy (LTEM) on the thin-plate specimens. CBED measurements can directly determine the crystal chirality, namely space group $P 4_{3} 32$ (defined as left-handed crystal with $\Gamma_{\mathrm{c}}=$ -1 ) or $P 4_{1} 32$ (defined as right-handed crystal with $\Gamma_{\mathrm{c}}=+1$ ). On the other hand, LTEM measurements can unambiguously identify the helicity of skyrmions (CCW or $\mathrm{CW}$ ), thereby allowing the determination of helicity of the corresponding helices, namely left-handed helix $\left(\gamma_{\mathrm{m}}=-1\right)$ or right-handed helix $\left(\gamma_{\mathrm{m}}=+1\right)$ as shown in Figs. 1(c)-1(f). Here, when $\boldsymbol{q}$ is parallel (antiparallel) to $\boldsymbol{m}_{1} \times \boldsymbol{m}_{2}, \boldsymbol{m}_{1}$ and $\boldsymbol{m}_{2}$ being magnetic moments in order along $\boldsymbol{q}$, the magnetic helicity is defined as right handed (left handed). As discussed in previous studies [16-22], the sign of the DMI is represented by the correlation $\Gamma_{\mathrm{c}} \gamma_{\mathrm{m}}$ due to both crystal chirality and magnetic helicity. From these multiple experiments, we found that the DMI systematically varies with the change in band filling and its sign change occurs around $x_{\mathrm{c}} \sim 2.7$ in $\mathrm{Co}_{8-x} \mathrm{Fe}_{x} \mathrm{Zn}_{8} \mathrm{Mn}_{4}$. Finally, the experimental results were compared to the theoretical results based on $a b$ initio electronic structure calculation.

\section{METHODS}

\section{A. Sample preparation}

Bulk polycrystalline ingots of $\mathrm{Co}_{8-x} \mathrm{Fe}_{x} \mathrm{Zn}_{8} \mathrm{Mn}_{4}$, composed of randomly oriented single-crystalline grains, were synthesized by a melt growth method from pure metals of $\mathrm{Co}, \mathrm{Fe}, \mathrm{Zn}$, and $\mathrm{Mn}$ with nominal compositions. Phase purity with the $\beta$-Mn-type crystal structure was confirmed using 
powder x-ray diffraction [Supplemental Figs. S1(a-c) [38]]. Chemical compositions were examined by energy dispersive $\mathrm{x}$-ray (EDX) spectroscopy, and found to be homogeneous and close to nominal compositions up to $x=4.5$ [Supplemental Figs. S1(d-f) [38]]. Above $x=5$, an inhomogeneous distribution of the elements was observed. Thus the solubility limit of $\mathrm{Fe}$ is around $x=4.5$. Bulk polycrystalline ingots of $\mathrm{Co}_{8-x} \mathrm{Ni}_{x} \mathrm{Zn}_{8} \mathrm{Mn}_{4}$ and $\mathrm{Co}_{8-x} \mathrm{Ru}_{x} \mathrm{Zn}_{8} \mathrm{Mn}_{4}$ were synthesized with the same method, and the solubility limit is found to be $x=2$ for $\mathrm{Ni}$ and $x=2.5$ for $\mathrm{Ru}$, respectively. The bulk ingots were cut into a rectangular shape with a size of $\sim 2 \mathrm{~mm} \times 1 \mathrm{~mm} \times 1 \mathrm{~mm}$ for magnetization, ac susceptibility, and SANS measurements. Only for $\mathrm{Co}_{8} \mathrm{Zn}_{8} \mathrm{Mn}_{4}$, a singlecrystalline piece grown by the Bridgman method was used. For CBED and LTEM measurements for $\mathrm{Co}_{8} \mathrm{Zn}_{8} \mathrm{Mn}_{4}$ and $\mathrm{Co}_{3.5} \mathrm{Fe}_{4.5} \mathrm{Zn}_{8} \mathrm{Mn}_{4}$, single-grain and single-chirality thin-plate specimens with a thickness of $\sim 150 \mathrm{~nm}$ were prepared from bulk pieces by mechanical polishing and subsequent argon ion milling.

\section{B. Magnetization and ac magnetic susceptibility}

Magnetization and ac magnetic susceptibility measurements were performed using the vibrating sample magnetometer (VSM) mode and ac susceptibility measurement mode, respectively, of a superconducting quantum interference device magnetometer (MPMS3, Quantum Design). For the ac susceptibility measurement, an ac excitation field with $1 \mathrm{Oe}$ and $193 \mathrm{~Hz}$ was applied parallel to a static magnetic field.

\section{SANS}

SANS measurements were performed using the instrument SANS-I at the Paul Scherrer Institute, Switzerland. Neutrons with a wavelength of $12 \AA$ were collimated over $18 \mathrm{~m}$ before the sample. The scattered neutrons were counted by a twodimensional position-sensitive multidetector located $20 \mathrm{~m}$ behind the sample. The samples were mounted on aluminum plates and installed in a horizontal field cryomagnet. The cryomagnet was rotated (rocked) around the vertical axis in the range from $-10^{\circ}$ to $+10^{\circ}\left(1^{\circ}\right.$ step) in the magnetic field $(H) \perp$ beam geometry. The SANS images shown in Supplemental Fig. S2 [38] were obtained by summing multiple SANS measurements obtained over the above rocking scans. For $\mathrm{Co}_{8} \mathrm{Zn}_{8} \mathrm{Mn}_{4}$, the same SANS data as our previous measurement [35] were used.

\section{CBED and LTEM}

For determination of the crystal chirality, CBED measurements were performed at room temperature with a transmission electron microscope (JEM-2100F) at an acceleration voltage of $200 \mathrm{kV}$. Convergent-beam electrons were incident along the [120] direction and diffraction patterns containing the first-order Laue zone were obtained by a charge-coupled device camera. For the simulation of CBED patterns, the MBFIT software [39] was used.

For determination of the magnetic helicity, LTEM measurements were performed with the same specimen and the electron microscope as used in the CBED measurements. A magnetic field was applied perpendicular to the specimen plate and its magnitude was controlled by tuning the electric current of the objective lens. Magnetic contrasts of skyrmions were observed as convergences (bright contrast) or divergences (dark contrast) of the electron beam on the defocused image planes.

\section{E. Theoretical calculation}

Electronic structure calculations for a hypothetical model material, $\mathrm{Co}_{8} \mathrm{Zn}_{12}$, were performed within the generalized gradient approximation [40] in the framework of the density functional theory as implemented in the quantum-ESPRESSO code [41]. For defining the crystal geometry, the experimental structure of $\mathrm{Co}_{8} \mathrm{Zn}_{8} \mathrm{Mn}_{4}$ was adopted. The DMI was calculated through spin current [31], which was computed using interpolated electronic structures obtained by the Wannier function technique [42]. The size of the spin-orbit couplings was confirmed to be sufficiently small so that the spin current is determined by the first order of the spin-orbit coupling $[43,44]$. Carrier density dependence is discussed within the rigid-band picture.

\section{RESULTS}

\section{A. Variation of magnetic phase diagram}

First, we show the temperature $(T)$ dependence of magnetization $(M)$ for $\mathrm{Co}_{8} \mathrm{Zn}_{8} \mathrm{Mn}_{4}(x=0), \mathrm{Co}_{5} \mathrm{Fe}_{3} \mathrm{Zn}_{8} \mathrm{Mn}_{4}(x=$ 3 ), and $\mathrm{Co}_{3.5} \mathrm{Fe}_{4.5} \mathrm{Zn}_{8} \mathrm{Mn}_{4}(x=4.5)$ in Figs. 2(a)-2(c). For $x=0, M$ exhibits a rapid increase upon cooling due to a helimagnetic transition at $T_{\mathrm{c}} \sim 300 \mathrm{~K}$ and a gradual reduction upon cooling below $\sim 120 \mathrm{~K}$, the latter of which corresponds to an increase in helical $q$ as explained in the next section. A sharp drop of $M$ around $10 \mathrm{~K}$ observed only in the data taken in a field-warming process after a zero-field cooling (ZFC) is due to a reentrant spin-glass transition [36]. For $x=3$ with $T_{\mathrm{c}} \sim 230 \mathrm{~K}$, on the other hand, while a sharp change in $M$ due to a spin-glass transition is observed in the ZFC curve, the gradual decrease around $100 \mathrm{~K}$ is not. For $x=4.5$, the $M$ again shows a gradual reduction below $T_{\mathrm{c}} \sim 135 \mathrm{~K}$ on cooling. Magnetization data for all the measured samples are presented in Supplemental Fig. S3(a) [38], showing a systematic thermal variation with the change in $x$.

In Figs. 2(d)-2(f), the real part of ac susceptibility $\left(\chi^{\prime}\right)$ around $T_{\mathrm{c}}$ is plotted against $H$ for $x=0,3$, and 4.5. In $x=0$ and 4.5, the slope of $\chi^{\prime}$ at low fields is positive, and clear dip structures, corresponding to a SkX state, are observed near $T_{\mathrm{c}}$. In $x=3$, on the other hand, the slope of $\chi^{\prime}$ at low fields is negative, and no dip structure ascribable to a SkX state is discerned at any temperature.

$H-T$ phase diagrams based on $\chi^{\prime}$ for $x=0,3$, and 4.5 are shown in Figs. 2(g)-2(i), respectively, and those for all the measured samples in Supplemental Fig. S4 [38]. The equilibrium SkX phase near $T_{\mathrm{c}}$ systematically changes with increasing $x$; the SkX phase shrinks as $x$ is increased from 0 to 3 , completely disappears at $x=3$, then reappears and expands as $x$ is further increased from 3 to 4.5 . These results are reasonably understood if the zero field helimagnetic state is suppressed in favor of a near ferromagnetic state as $x$ approaches 3 . 


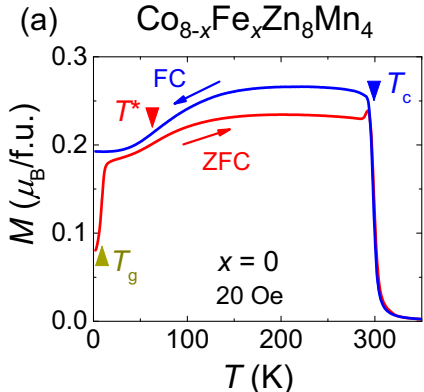

(d)
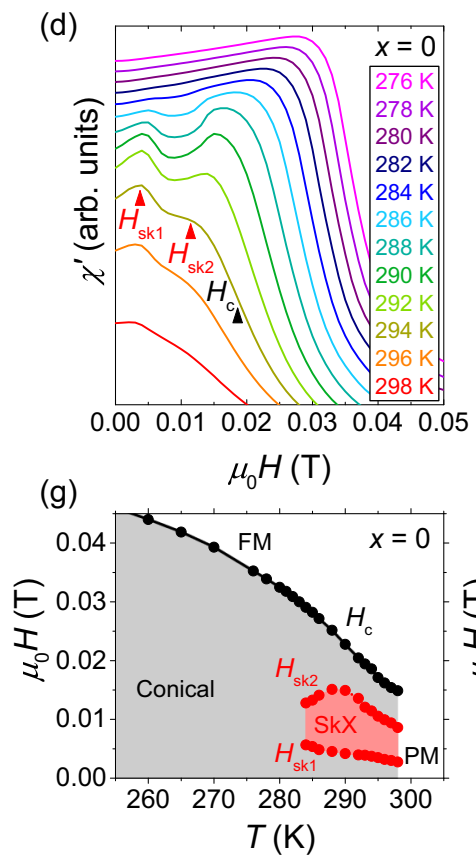

(b)

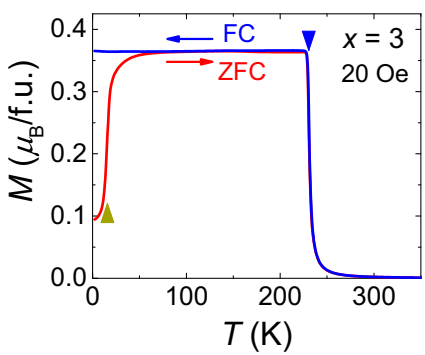

(e)

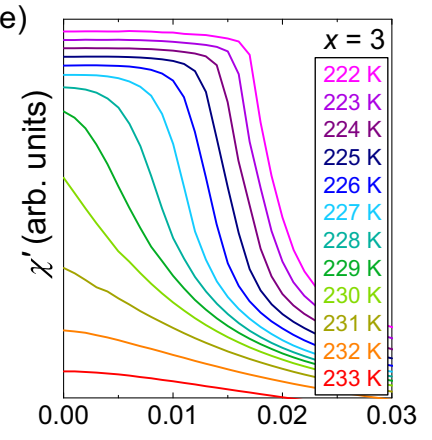

(h)

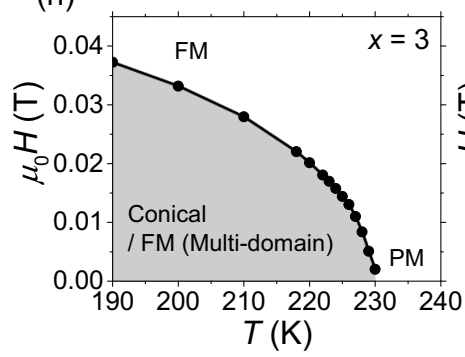

(c)

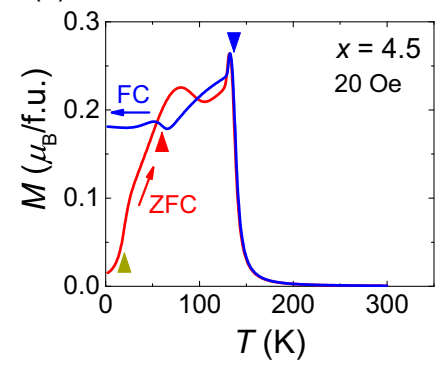

(f)

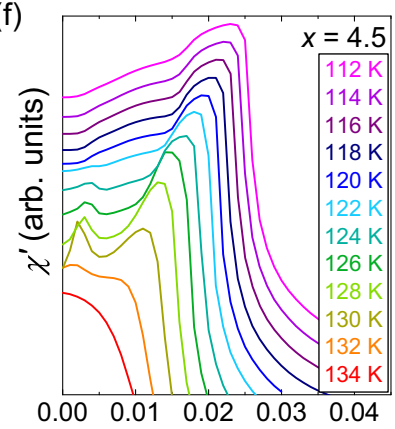

(i) $\quad \mu_{0} H(\mathrm{~T})$

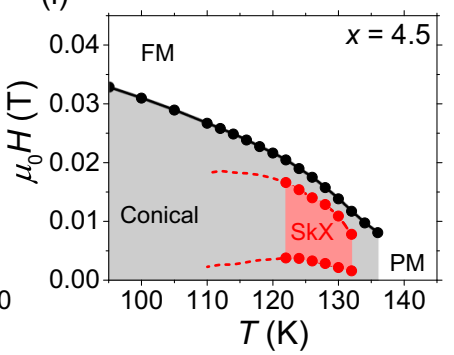

FIG. 2. (a)-(c) Temperature ( $T$ ) dependence of the magnetization $(M)$ under a magnetic field of 20 Oe for $x=0,3$, and 4.5 , respectively. A blue line shows the data taken in a field-cooling (FC) process and a red line the data collected in a field-warming process after zero-field cooling (ZFC) down to $2 \mathrm{~K}$. A helimagnetic transition temperature $T_{\mathrm{c}}$ (blue triangle) is determined as an inflection point of the steep increase in $M$ on cooling. A reentrant spin-glass transition temperature $T_{\mathrm{g}}$ (yellow triangle) is defined as an inflection point of the sharp increase in $M$ in the warming process after ZFC. $T^{*} \sim 60 \mathrm{~K}$ indicated with red triangles in (a) and (c) corresponds to an inflection point of gradual decrease in $M$ on cooling below $120 \mathrm{~K}$, which is hardly observed for $x=3$ in (b). (d)-(f) Magnetic field $(H)$ dependence of the real part of the ac magnetic susceptibility $\left(\chi^{\prime}\right)$ in a field-decreasing process from an induced-ferromagnetic region at several temperatures near $T_{\mathrm{c}}$ for $x=0,3$, and 4.5 , respectively. The phase boundaries between conical and SkX states $\left[H_{\mathrm{sk} 1}\right.$ and $H_{\mathrm{sk} 2}$, red triangles in (d)] were determined as peak positions of $\chi^{\prime}$. The phase boundary between conical and induced-ferromagnetic states $\left[H_{\mathrm{c}}\right.$, black triangle in (d)] was determined as an inflection point of $\chi^{\prime}$. In $x=4.5$, signatures of the SkX phase become unclear below $120 \mathrm{~K}$, but some anomalies are still discerned, implying a reduced volume fraction of skyrmions. (g)-(i) $H-T$ phase diagram determined by $\chi^{\prime}$ for $x=0,3$, and 4.5, respectively. In $x=3$, the magnetic state at low fields is either a conical state with a very long periodicity or a ferromagnetic multidomain state. In $x=4.5$, the blurred boundaries of the skyrmion phase below $120 \mathrm{~K}$ are plotted with dotted red lines.

\section{B. Determination of helical wave vectors}

To determine helical periodicity in $\mathrm{Co}_{8-x} \mathrm{Fe}_{x} \mathrm{Zn}_{8} \mathrm{Mn}_{4}$, we performed SANS measurements. As displayed in Supplemental Fig. S2 [38], clear Bragg spots described by helical wave vectors $\boldsymbol{q}$ were observed in the $q_{x}-q_{y}$ plane below $T_{\mathrm{c}}$ at $H=0$. Figure 3(a) shows azimuthal-angle-averaged SANS intensity as a function of $|\boldsymbol{q}|(=q)$ for all the measured samples at $150 \mathrm{~K}$ $(120 \mathrm{~K}$ for $x=4.5)$ and $50 \mathrm{~K}(40 \mathrm{~K}$ for $x=0)$. At $150 \mathrm{~K}$ (or $120 \mathrm{~K}$ ), as $x$ is increased from 0 to 3 , the Bragg peak systematically shifts to lower $q$, and then back to higher $q$ as $x$ is further increased from 3 to 4.5 . For $x=3$, we observed no peak structure within the detectable $q$ range $\left(0.01 \mathrm{~nm}^{-1}<\right.$ $q<0.14 \mathrm{~nm}^{-1}$ ), but only the tail of a peak centered at $q \sim 0$.
By fitting the peaks to a Gaussian function and plotting the peak center against $x$ as a helical $q$ in Fig. 3(b), we observe clearly a characteristic V-shaped curve as previously reported for $\mathrm{Mn}_{1-x} \mathrm{Fe}_{x} \mathrm{Ge}$ [19] and $\mathrm{Fe}_{1-x} \mathrm{Co}_{x} \mathrm{Ge}$ [21]. On the basis of the available data points, the $q$ value is expected to be zero at $x_{\mathrm{c}} \sim 2.7$. The helical periodicity $\lambda$ is also plotted against $x$ in Fig. 3(b), confirming the divergent behavior around $x_{\mathrm{c}}$.

As displayed in Fig. 3(a), as the temperature is lowered to $50 \mathrm{~K}$ the Bragg peaks shift to higher $q$ and become weaker and broader than at $150 \mathrm{~K}$ for all $x$, except for $x=3$. The detailed temperature dependence of helical $q$ is shown in Supplemental Fig. S3(b) [38]. A comparison between $M$ and 

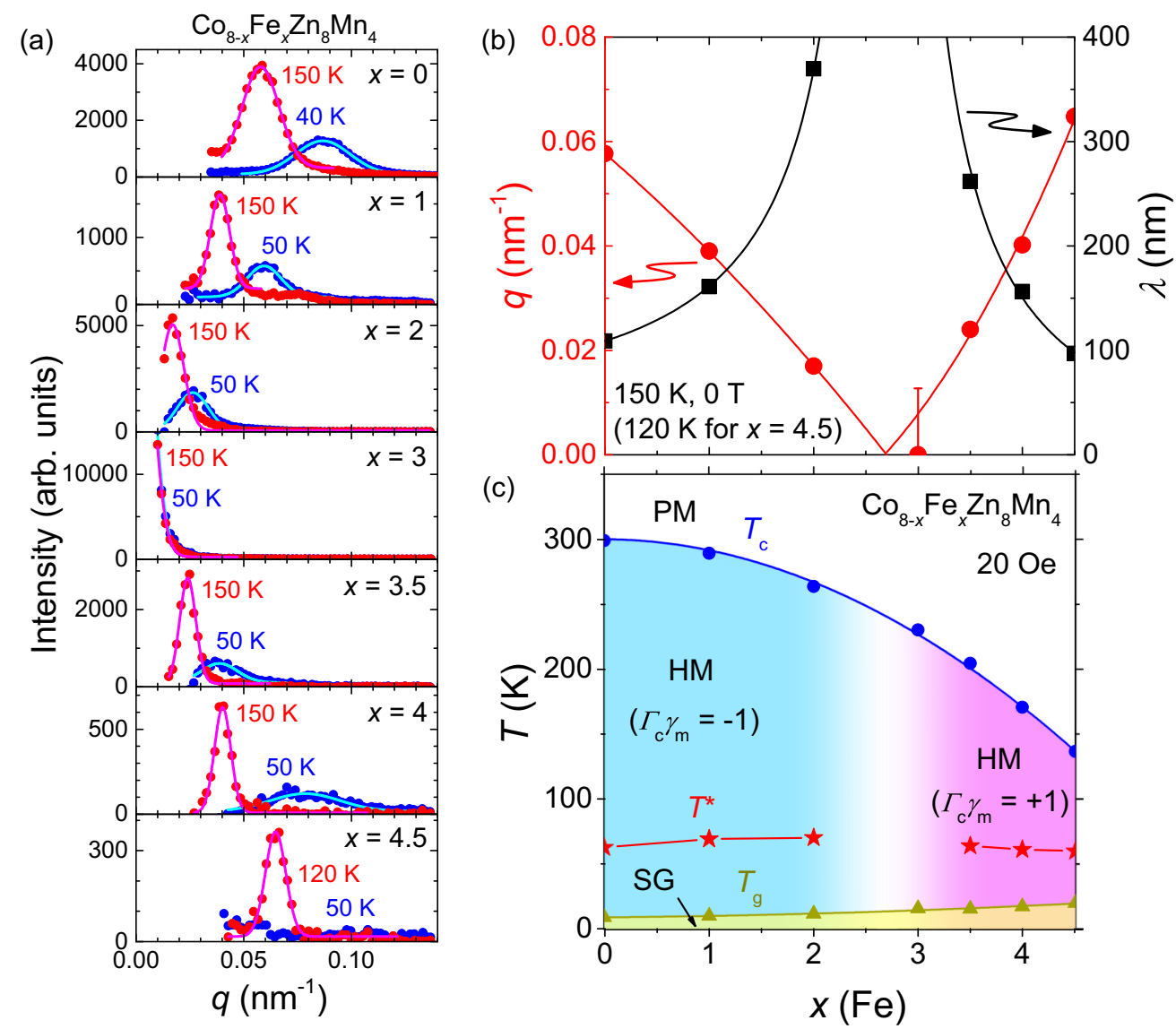

FIG. 3. (a) Small-angle neutron scattering (SANS) intensity as a function of $|\boldsymbol{q}|(=q)$ for all the measured samples at $150 \mathrm{~K}$ (120 K for $x=4.5$, red circles) and $50 \mathrm{~K}$ (40 K for $x=0$, blue circles) under zero magnetic field. These SANS intensities are obtained by averaging over a full azimuthal-angle range of $360^{\circ}$ around the origin of the two-dimensional SANS patterns (Supplemental Fig. S2 [38]). Data points are fitted to a Gaussian function denoted with pink lines for $150 \mathrm{~K}(120 \mathrm{~K}$ for $x=4.5)$ and light-blue lines for $50 \mathrm{~K}(40 \mathrm{~K}$ for $x=0)$. (b) $x$ dependence of helical $q$ (red circles) at $150 \mathrm{~K}(120 \mathrm{~K}$ for $x=4.5)$ determined as a peak position of a Gaussian function in (a). For $x=3$, an error bar is appended as the width of the Gaussian function centered at $q=0$. By interpolating the data points (red lines), $q$ is expected to be zero at $x_{\mathrm{c}} \sim 2.7$. The helical periodicity $\lambda=2 \pi / q$ is also plotted with black squares to show the divergent behavior around $x_{\mathrm{c}}$. (c) $T$ - $x$ phase diagram determined by magnetization measurements at 20 Oe [see Figs. 2(a)-2(c) and Supplemental Fig. S3(a) [38] for details]. The paramagnetic (PM)-helimagnetic (HM) transition temperature $T_{\mathrm{c}}$ is shown by blue circles, and the spin glass (SG) transition temperature $T_{\mathrm{g}}$ is denoted by yellow triangles. $T^{*}$ (red asterisks) indicates a temperature corresponding to the inflection point during a gradual reduction in $M$ on cooling, and also to an increase in helical $q$ (see Supplemental Fig. S3 [38]). Two helimagnetic phases (light-blue and pink areas) separated by a nearly ferromagnetic region (white area below $T_{\mathrm{c}}$ ) are characterized by opposite signs of $\Gamma_{\mathrm{c}} \gamma_{\mathrm{m}}$.

$q$ indicates that the gradual reduction of $M$ below $120 \mathrm{~K}$ correlates with the increase in $q$, except for $x=3$.

By applying a transverse magnetic field, the positions of the Bragg spots are switched so that their wave vectors become aligned with the field direction, which corresponds to the transition from a helical multidomain state $(\boldsymbol{q} \|\langle 100\rangle)$ to a conical state $(\boldsymbol{q} \| H)$ (Supplemental Fig. S2 [38]). Note that, for $x=3$, Bragg spots are never observed at any temperatures and any magnetic fields in the detectable $q$ range in this transverse configuration. These SANS results confirm that the magnetic state in $x=3$ is nearly ferromagnetic rather than helimagnetic, in good agreement with expectation according to the results of magnetization measurements.

The $T-x$ phase diagram determined from the magnetization and SANS measurements is shown in Fig. 3(c). The magnetic phase below $T_{\mathrm{c}}$ can be described as consisting of two helimagnetic regions at $0 \leqslant x<x_{\mathrm{c}}$ and $x_{\mathrm{c}}<x \leqslant 4.5$ and a nearly ferromagnetic region at $x_{\mathrm{c}} \sim 2.7$ in between. The structure of this phase diagram is consistent with the occurrence of a sign change in DMI across $x_{\mathrm{c}}$ as demonstrated directly in the next section.

\section{Determination of crystal chirality and magnetic helicity}

To determine crystal chirality and magnetic helicity, we performed CBED and LTEM measurements for thin-plate specimens with two compositions, $x=0$ and 4.5. Figures 4(a), 4(b) and 4(e), 4(f) show the experimentally obtained CBED patterns for $x=0$ and 4.5, while simulated CBED patterns for the $\beta$-Mn-type structure with $P 4_{1} 32$ and $P 4_{3} 32$ are presented in Figs. 4(c), 4(d) and 4(g), 4(h), respectively. The experimental pattern for $x=0$ agrees well with the simulated one for $P 4_{1} 32$, while that for $x=4.5$ is in good accord with $P_{4} 32$, indicating that the crystal chirality of 


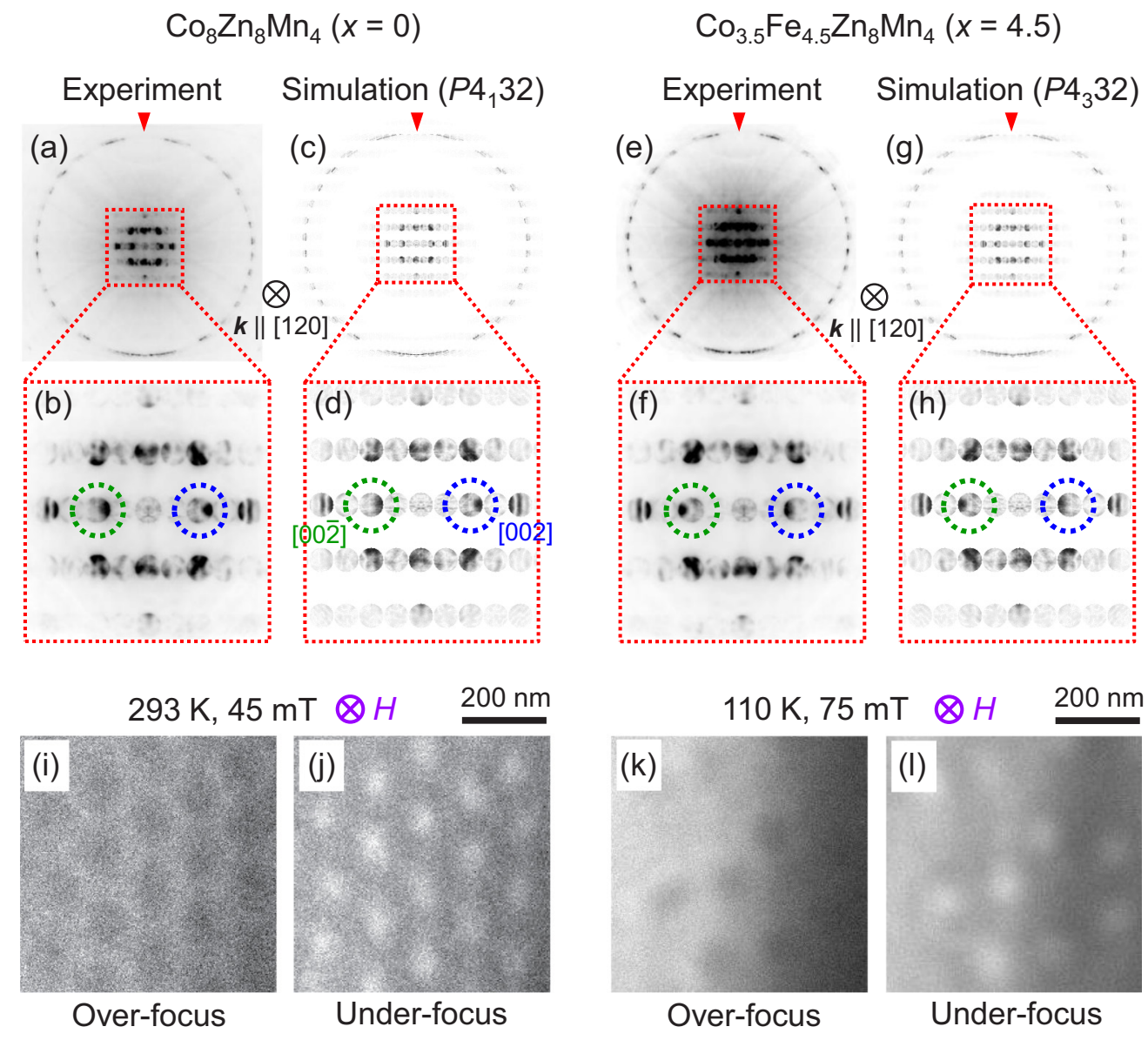

FIG. 4. (a),(b) Convergent-beam electron diffraction (CBED) patterns observed from a thin-plate $\operatorname{specimen}$ of $\mathrm{Co}_{8} \mathrm{Zn}_{8} \mathrm{Mn}_{4}$. (c),(d) Simulated CBED patterns for right-handed crystal with the space group of $P 4_{1} 32$. (e),(f) CBED patterns observed in a thin-plate specimen of $\mathrm{Co}_{3.5} \mathrm{Fe}_{4.5} \mathrm{Zn}_{8} \mathrm{Mn}_{4}$. (g),(h) Simulated CBED patterns for left-handed crystal with the space group of $P 4_{3} 32$. For all the panels (a)-(h), the incident electron beam direction is along the [120] direction. A red triangle in upper panels (a), (c), (e), and (g) indicates the disconnected intensity at the top part of circular diffraction that makes a distinction with the connected intensity at the bottom part, reflecting broken inversion symmetry. Lower panels (b), (d), (f), and (h) are expanded images for the red dotted-line enclosed areas of (a), (c), (e), and (g), respectively. Blue and green dotted circles in (b), (d), (f), and (h) highlight [002] and [00ㄹ] diffraction signals, respectively. (i)-(l) Lorentz transmission electron microscope (LTEM) images observed for the same thin-plate specimens as used in the CBED measurements. Panel (i)[(j)] is an over(under-) focused LTEM image for $\mathrm{Co}_{8} \mathrm{Zn}_{8} \mathrm{Mn}_{4}$, measured at $293 \mathrm{~K}$ and under a magnetic field of $45 \mathrm{mT}$ applied perpendicular (into the page) to the thin specimen. Panel (k)[(1)] is an over- (under-) focused LTEM image for $\mathrm{Co}_{3.5} \mathrm{Fe}_{4.5} \mathrm{Zn}_{8} \mathrm{Mn}_{4}$, measured at $110 \mathrm{~K}$ and $75 \mathrm{mT}$.

the examined specimens are right handed and left handed for $x=0$ and 4.5 , respectively.

Next we present real-space images of skyrmions obtained by LTEM observations for exactly the same specimens as used in the CBED measurements. Figure 4(i) [4(j)] shows the over(under-) focused LTEM image observed for $x=0$ at $293 \mathrm{~K}$ and $45 \mathrm{mT}$, in which skyrmions with a diameter of $\sim 140 \mathrm{~nm}$ are clearly observed to form a triangular lattice. The observed dark (bright) dots in the over- (under-) focused image indicate that the helicity of the skyrmions is CCW consistent with the illustration shown in Fig. 1(e).

The over- (under-) focused LTEM image observed for $x=4.5$ at $110 \mathrm{~K}$ and $75 \mathrm{mT}$ is displayed in Fig. 4(k)[4(1)]. Skyrmions with a diameter of $\sim 130 \mathrm{~nm}$ are discerned and their arrangement is consistent with a finite positional disorder. The helicity of the skyrmions is CCW, the same as $x=0$, as evidenced by dark (bright) dots in the over- (under-) focused image.
In Table I, the experimentally determined crystal chirality $\Gamma_{\mathrm{c}}$, magnetic helicity $\gamma_{\mathrm{m}}$, and their correlation $\Gamma_{\mathrm{c}} \gamma_{\mathrm{m}}$ for $x=0$ and 4.5 are summarized. While the crystal chiralities in the examined samples of $x=0$ and 4.5 are opposite to each other, the helicity of the skyrmions is the same, resulting in the opposite sign of the chirality-helicity correlation: $\Gamma_{\mathrm{c}} \gamma_{\mathrm{m}}=-1$ for $x=0$ and $\Gamma_{\mathrm{c}} \gamma_{\mathrm{m}}=+1$ for $x=4.5$. Thus it is concluded that the two helimagnetic phases shown in Fig. 3(c) possess

TABLE I. Crystal chirality $\Gamma_{\mathrm{c}}$, magnetic helicity $\gamma_{\mathrm{m}}$, and their correlation $\Gamma_{\mathrm{c}} \gamma_{\mathrm{m}}$ for $\mathrm{Co}_{8} \mathrm{Zn}_{8} \mathrm{Mn}_{4}$ and $\mathrm{Co}_{3.5} \mathrm{Fe}_{4.5} \mathrm{Zn}_{8} \mathrm{Mn}_{4}$ determined by CBED and LTEM measurements.

\begin{tabular}{lccc}
\hline \hline & $\Gamma_{\mathrm{c}}$ & $\gamma_{\mathrm{m}}$ & $\Gamma_{\mathrm{c}} \gamma_{\mathrm{m}}$ \\
\hline $\mathrm{Co}_{8} \mathrm{Zn}_{8} \mathrm{Mn}_{4}$ & +1 & -1 & -1 \\
$\mathrm{Co}_{3.5} \mathrm{Fe}_{4.5} \mathrm{Zn}_{8} \mathrm{Mn}_{4}$ & -1 & -1 & +1 \\
\hline \hline
\end{tabular}



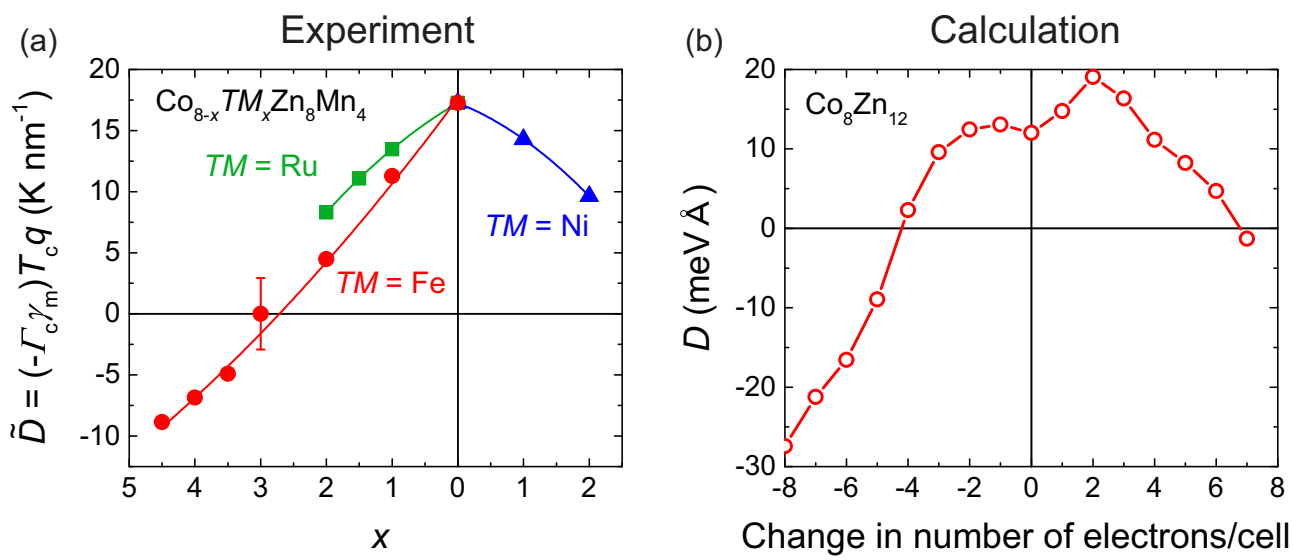

FIG. 5. (a) Dopant concentration (x) dependence of $\tilde{D} \equiv\left(-\Gamma_{\mathrm{c}} \gamma_{\mathrm{m}}\right) T_{\mathrm{c}} q$, which is proportional to Dzyaloshinskii-Moriya interaction $D$, in $\mathrm{Co}_{8-x} T M_{x} \mathrm{Zn}_{8} \mathrm{Mn}_{4}$. Here, $T M=\mathrm{Fe}$ (red circles) and $\mathrm{Ru}$ (green squares) correspond to hole doping, while $T M=\mathrm{Ni}$ (blue triangles) to electron doping. The values of $T_{\mathrm{c}}$ and $q$ are determined by magnetization and SANS measurements, respectively. See Supplemental Figs. S5 and S6 [38] for details of magnetization and SANS measurements for $T M=\mathrm{Ni}$ and $\mathrm{Ru}$. The minus sign is appended to the definition of $\tilde{D}$ due to the following reason: both the experiment and theory consistently show that the right-handed crystal $\left(\Gamma_{\mathrm{c}}=+1\right)$ of the Fe nondoped material exhibits a left-handed helix $\left(\gamma_{\mathrm{m}}=-1\right)$. In our theoretical definition, this corresponds to $D>0$; therefore, we included the minus sign to keep the consistency. (b) Theoretically calculated value of $D$ plotted as a function of electron number per unit cell, which is taken as the origin for a hypothetical model compound $\mathrm{Co}_{8} \mathrm{Zn}_{12}$, where $8 c$ and $12 d$ sites are occupied by only Co and $\mathrm{Zn}$, respectively. The calculated band structure and density of states are shown in Supplemental Fig. S7 [38].

opposite sign of $\Gamma_{\mathrm{c}} \gamma_{\mathrm{m}}$ and the sign reversal occurs at $x_{\mathrm{c}} \sim$ 2.7. Therefore, if the crystal chirality is fixed, the helicity of the magnetic helices and skyrmions changes sign when $x$ crosses $x_{\mathrm{c}}$.

\section{Variation of DMI}

In Fig. 5, we show the variation in DMI as a function of electron filling obtained from experiments and theoretical calculations. In general, the helimagnetic transition temperature $T_{\mathrm{c}}$ is approximately proportional to $J$ when $J \gg D$ like the present case, and the helical $q$ is given by $q \propto D / J$. Thus we plot $\tilde{D} \equiv\left(-\Gamma_{\mathrm{c}} \gamma_{\mathrm{m}}\right) T_{\mathrm{c}} q$ as a measure of $D$ in Fig. 5(a). For $T_{\mathrm{c}}$ and $q$, values determined from the magnetization and SANS data are used, respectively. In addition to the data for $\mathrm{Co}_{8-x} \mathrm{Fe}_{x} \mathrm{Zn}_{8} \mathrm{Mn}_{4}$, those for $\mathrm{Co}_{8-x} \mathrm{Ni}_{x} \mathrm{Zn}_{8} \mathrm{Mn}_{4}$ and $\mathrm{Co}_{8-x} \mathrm{Ru}_{x} \mathrm{Zn}_{8} \mathrm{Mn}_{4}$ are also plotted (see Supplemental Fig. S6 [38] for $T_{\mathrm{c}}$ and $q$ values in Ni- and Ru-doped systems). In $\mathrm{Co}_{8-x} \mathrm{Fe}_{x} \mathrm{Zn}_{8} \mathrm{Mn}_{4}, \tilde{D}$ significantly decreases as $x$ is increased, or equivalently as the electron filling is decreased, and the sign reversal of $\tilde{D}$ occurs at $x_{\mathrm{c}} \sim 2.7$. For both $\mathrm{Ni}$ and $\mathrm{Ru}$ doping, on the other hand, $\tilde{D}$ decreases more gradually and the sign reversal is not observed within the experimentally available concentration range $(x \leqslant 2)$. Although $\mathrm{Ru}$ doping would be identical to $\mathrm{Fe}$ doping in terms of band filling in the case of fully amalgamated bands of $3 d$ (Co) and $4 d$ $(\mathrm{Ru})$ electron states, or would be expected to give rise to even larger effect than Fe doping due to stronger spin-orbit interaction, the experimentally observed change in $\tilde{D}$ with $\mathrm{Ru}$ doping is smaller than with $\mathrm{Fe}$ doping. This is probably because Ru substitution makes exchange splitting smaller, as expected from the rapid suppression of $T_{\mathrm{c}}$ and the saturation magnetization (Supplemental Fig. S6 [38]), and hence alters the electronic structure in addition to the change in electron density and atomic spin-orbit interaction.
To evaluate the DMI theoretically, we performed $a b$ initio electronic structure calculations for a hypothetical model material $\mathrm{Co}_{8} \mathrm{Zn}_{12}$ [32], where $8 c$ and $12 d$ sites are assumed to be fully occupied by Co and $\mathrm{Zn}$, respectively. As shown in Supplemental Fig. S7 [38], the relevant states near the Fermi level are mainly due to the Co-3d band. By using the spin-current formula described in Ref. [31], $D$ is theoretically calculated and plotted as a function of electron band filling in Fig. 5(b). As the carrier density falls, which corresponds to the partial substitution of $\mathrm{Fe}$ for $\mathrm{Co}, D$ decreases and its sign reversal occurs at around -4 electron/cell. Upon increasing the carrier density, which corresponds to the partial substitution of $\mathrm{Ni}$ for $\mathrm{Co}$, the sign reversal of $D$ also occurs, but at as large as +7 electron/cell. These theoretical results qualitatively reproduce the experimental results presented in Fig. 5(a). Therefore, the observed variation of the DMI in $\mathrm{Co}_{8-x} T M_{x} \mathrm{Zn}_{8} \mathrm{Mn}_{4}$, and its sign reversal in the case of $T M=\mathrm{Fe}$, can be understood in terms of the compositional tuning of the band filling, or equivalently in terms of the chemical potential within the complex band structure.

\section{CONCLUSION}

In $\mathrm{Co}_{8-x} \mathrm{Fe}_{x} \mathrm{Zn}_{8} \mathrm{Mn}_{4}(0 \leqslant x \leqslant 4.5)$ the strength of the DMI decreases as the Fe doping proceeds, and its sign change occurs at a critical $\mathrm{Fe}$ concentration $x_{\mathrm{c}} \sim 2.7$. Upon further Fe doping, the magnitude of the DMI increases again. This gives rise to a reversal of the helicity of magnetic helices and skyrmions through the nearly ferromagnetic state at $x_{\mathrm{c}}$ under a fixed crystal chirality. The experimental results are well explained in terms of the change in $3 d$ band filling by the theory using a spin-current formulation and first-principles electronic structure calculation. Band anticrossing points near the Fermi level within a complex band structure likely play an important 
role on these observations, as emphasized in Ref. [30]. The present study demonstrates that the DMI is controlled by the change in band filling and that in principle the sign reversal of DMI universally occurs in metallic chiral magnets with easily tunable band filling. Our observations provide a promising route for tailoring both the size and helicity of skyrmions and may inspire novel ideas for applications based on designed compositional gradients and that enable the local control of the DMI strength at the nanoscale.

\section{ACKNOWLEDGMENTS}

We are grateful to A. Kikkawa for supports of sample preparation. This work was supported by the Japan Society for the Promotion of Science (JSPS) Grants-in-Aid for Scientific Research (S) No. 24224009 and for Young Scientists (B) No. 17K18355, the Swiss National Science Foundation (SNSF) Sinergia network "NanoSkyrmionics", the SNSF Projects No. 153451 and No. 166298, and the European Research Council project CONQUEST.
[1] A. N. Bogdanov and D. A. Yablonskii, Sov. Phys. JETP 68, 101 (1989).

[2] N. Nagaosa and Y. Tokura, Nat. Nanotechnol. 8, 899 (2013).

[3] S. Mühlbauer, B. Binz, F. Jonietz, C. Pfleiderer, A. Rosch, A. Neubauer, R. Georgii, and P. Böni, Science 323, 915 (2009).

[4] X. Z. Yu, Y. Onose, N. Kanazawa, J. H. Park, J. H. Han, Y. Matsui, N. Nagaosa, and Y. Tokura, Nature (London) 465, 901 (2010).

[5] P. Milde, D. Köhler, J. Seidel, L. M. Eng, A. Bauer, A. Chacon, J. Kindervater, S. Mühlbauer, C. Pfleiderer, S. Buhrandt, C. Schütte, and A. Rosch, Science 340, 1076 (2013).

[6] N. Kanazawa, J.-H. Kim, D. S. Inosov, J. S. White, N. Egetenmeyer, J. L. Gavilano, S. Ishiwata, Y. Onose, T. Arima, B. Keimer, and Y. Tokura, Phys. Rev. B 86, 134425 (2012).

[7] T. Tanigaki, K. Shibata, N. Kanazawa, X. Z. Yu, Y. Onose, H. S. Park, D. Shindo, and Y. Tokura, Nano Lett. 15, 5438 (2015).

[8] X. Z. Yu, N. Kanazawa, Y. Onose, K. Kimoto, W. Z. Zhang, S. Ishiwata, Y. Matsui, and Y. Tokura, Nat. Mater. 10, 106 (2011).

[9] C. Pappas, L. J. Bannenberg, E. Lelievre-Berna, F. Qian, C. D. Dewhurst, R. M. Dalgliesh, D. L. Schlagel, T. A. Lograsso, and P. Falus, Phys. Rev. Lett. 119, 047203 (2017).

[10] F. Qian, H. Wilhelm, A. Aqeel, T. T. M. Palstra, A. J. E. Lefering, E. H. Brück, and C. Pappas, Phys. Rev. B 94, 064418 (2016)

[11] S. Pöllath, J. Wild, L. Heinen, T. N. G. Meier, M. Kronseder, L. Tutsch, A. Bauer, H. Berger, C. Pfleiderer, J. Zweck, A. Rosch, and C. H. Back, Phys. Rev. Lett. 118, 207205 (2017).

[12] F. Jonietz, S. Muhlbauer, C. Pfleiderer, A. Neubauer, W. Münzer, A. Bauer, T. Adams, R. Georgii, P. Böni, R. A. Duine, K. Everschor, M. Garst, and A. Rosch, Science 330, 1648 (2010).

[13] T. Schulz, R. Ritz, A. Bauer, M. Halder, M. Wagner, C. Franz, C. Pfleiderer, K. Everschor, M. Garst, and A. Rosch, Nat. Phys. 8, 301 (2012).

[14] J. Iwasaki, M. Mochizuki, and N. Nagaosa, Nat. Nanotechnol. 8, 742 (2013).

[15] J. Sampaio, V. Cros, S. Rohart, A. Thiaville, and A. Fert, Nat. Nanotechnol. 8, 839 (2013).

[16] S. V. Grigoriev, D. Chernyshov, V. A. Dyadkin, V. Dmitriev, E. V. Moskvin, D. Lamago, Th. Wolf, D. Menzel, J. Schoenes, S. V. Maleyev, and H. Eckerlebe, Phys. Rev. B 81, 012408 (2010)

[17] S. V. Grigoriev, D. Chernyshov, V. A. Dyadkin, V. Dmitriev, S. V. Maleyev, E. V. Moskvin, D. Menzel, J. Schoenes, and H. Eckerlebe, Phys. Rev. Lett. 102, 037204 (2009).

[18] D. Morikawa, K. Shibata, N. Kanazawa, X. Z. Yu, and Y. Tokura, Phys. Rev. B 88, 024408 (2013).
[19] S. V. Grigoriev, N. M. Potapova, S.-A. Siegfried, V. A Dyadkin, E. V. Moskvin, V. Dmitriev, D. Menzel, C. D. Dewhurst, D. Chernyshov, R. A. Sadykov, L. N. Fomicheva, and A. V. Tsvyashchenko, Phys. Rev. Lett. 110, 207201 (2013).

[20] K. Shibata, X. Z. Yu, T. Hara, D. Morikawa, N. Kanazawa, K. Kimoto, S. Ishiwata, Y. Matsui, and Y. Tokura, Nat. Nanotechnol. 8, 723 (2013).

[21] S. V. Grigoriev, S.-A. Siegfried, E. V. Altynbayev, N. M. Potapova, V. Dyadkin, E. V. Moskvin, D. Menzel, A. Heinemann, S. N. Axenov, L. N. Fomicheva, and A. V. Tsvyashchenko, Phys. Rev. B 90, 174414 (2014).

[22] V. Dyadkin, K. Prša, S. V. Grigoriev, J. S. White, P. Huang, H. M. Rønnow, A. Magrez, C. D. Dewhurst, and D. Chernyshov, Phys. Rev. B 89, 140409(R) (2014).

[23] M. Heide, G. Bihlmayer, and S. Blügel, Phys. Rev. B 78, 140403(R) (2008).

[24] M. Heide, G. Bihlmayer, and S. Blügel, Physica B: Phys. Condens. Matter 404, 2678 (2009).

[25] J. Gayles, F. Freimuth, T. Schena, G. Lani, P. Mavropoulos, R. A. Duine, S. Blügel, J. Sinova, and Y. Mokrousov, Phys. Rev. Lett. 115, 036602 (2015).

[26] M. I. Katsnelson, Y. O. Kvashnin, V. V. Mazurenko, and A. I. Lichtenstein, Phys. Rev. B 82, 100403(R) (2010).

[27] F. Freimuth, R. Bamler, Y. Mokrousov, and A. Rosch, Phys. Rev. B 88, 214409 (2013).

[28] F. Freimuth, S. Blügel, and Y. Mokrousov, J. Phys.: Condens. Matter 26, 104202 (2014).

[29] O. Janson, I. Rousochatzakis, A. A. Tsirlin, M. Belesi, A. A. Leonov, U. K. Rößler, J. van den Brink, and H. Rosner, Nat. Commun. 5, 5376 (2014).

[30] T. Koretsune, N. Nagaosa, and R. Arita, Sci. Rep. 5, 13302 (2015).

[31] T. Kikuchi, T. Koretsune, R. Arita, and G. Tatara, Phys. Rev. Lett. 116, 247201 (2016).

[32] W. Xie, S. Thimmaiah, J. Lamsal, J. Liu, T. W. Heitmann, D. Quirinale, A. I. Goldman, V. Pecharsky, and G. J. Miller, Inorg. Chem. 52, 9399 (2013).

[33] T. Hori, H. Shiraish, and Y. Ishii, J. Magn. Magn. Mater. 310, 1820 (2007).

[34] Y. Tokunaga, X. Z. Yu, J. S. White, H. M. Rønnow, D. Morikawa, Y. Taguchi, and Y. Tokura, Nat. Commun. 6, 7638 (2015).

[35] K. Karube, J. S. White, N. Reynolds, J. L. Gavilano, H. Oike, A. Kikkawa, F. Kagawa, Y. Tokunaga, H. M. Rønnow, Y. Tokura, and Y. Taguchi, Nat. Mater. 15, 1237 (2016).

[36] K. Karube, J. S. White, D. Morikawa, C. D. Dewhurst, R. Cubitt, A. Kikkawa, X. Z. Yu, Y. Tokunaga, T. Arima, H. M. 
Rønnow, Y. Tokura, and Y. Taguchi, Sci. Adv. 4, eaar7043 (2018).

[37] R. Takagi, D. Morikawa, K. Karube, N. Kanazawa, K. Shibata, G. Tatara, Y. Tokunaga, T. Arima, Y. Taguchi, Y. Tokura, and S. Seki, Phys. Rev. B 95, 220406(R) (2017).

[38] See Supplemental Material at http://link.aps.org/supplemental/ 10.1103/PhysRevB.98.155120 for details.

[39] K. Tsuda and M. Tanaka, Acta Crystallogr., A 55, 939 (1999).

[40] J. P. Perdew, K. Burke, and M. Ernzerhof, Phys. Rev. Lett. 77, 3865 (1996).

[41] P. Giannozzi, S. Baroni, N. Bonini, M. Calandra, R. Car, C. Cavazzoni, D. Ceresoli, G. L. Chiarotti, M. Cococcioni, I. Dabo, A. Dal Corso, S. Fabris, G. Fratesi, S. de Gironcoli,
R. Gebauer, U. Gerstmann, C. Gougoussis, A. Kokalj, M. Lazzeri, L. Martin-Samos, N. Marzari, F. Mauri, R. Mazzarello, S. Paolini, A. Pasquarello, L. Paulatto, C. Sbraccia, S. Scandolo, G. Sclauzero, A. P. Seitsonen, A. Smogunov, P. Umari, and R. M. Wentzcovitch, J. Phys.: Condens. Matter 21, 395502 (2009).

[42] A. A. Mostofi, J. R. Yates, Y.-S. Lee, I. Souza, D. Vanderbilt, and N. Marzari, Comput. Phys. Commun. 178, 685 (2008).

[43] F. Freimuth, S. Blügel, and Y. Mokrousov, Phys. Rev. B 96, 054403 (2017).

[44] T. Koretsune, T. Kikuchi, and R. Arita, J. Phys. Soc. Jpn. 87, 041011 (2018). 